\title{
Novel synthetic nucleotides of notifiable dengue (1-4), Japanese encephalitis, yellow fever and Zika flaviviruses
}

\author{
Gerry Amor Camer ${ }^{1}$, Yuki Oikawa², Hitomi Omaki \& Daiji Endoh*,2 \\ ${ }^{1}$ University of Eastern Philippines, Department of Clinical Sciences - College of Veterinary Medicine, Catarman, Northern Samar \\ 6400 Philippines \\ ${ }^{2}$ Rakuno Gakuen University, Department of Radiation Biology-School of Veterinary Medicine, Ebetsu, 069-8501 Hokkaido, Japan \\ *Author for correspondence: Tel.: +81 11388 4847; Fax: +81 11387 5890; dendoh@rakuno.ac.jp
}

\begin{abstract}
Aim: To produce synthetic nucleotides of notifiable dengue virus (1-4 types), Japanese encephalitis, yellow fever and Zika flaviviruses. These notifiable flaviviruses, particularly dengue and Zika, are problematic mosquito-borne infections in the Philippines, as well as in those countries with tropical and subtropical climates. Method: An algorithmic design formulation of overlap extension - polymerase chain reaction (OE-PCR) was performed to propagate 50-60 oligomer lengths of select notifiable flaviviral RNAs to DNA nucleotides via the two-step process of OE-PCR. Result: Algorithmic OE-PCR design formulation efficiently produced 253-256 bp of notifiable flaviviruses. Comparing the newly designed algorithmic OE-PCR with existing executable programs demonstrated it to be efficient and useful in generating accurate sequences of synthetic flaviviral nucleotides. Conclusion: The efficiently and accurately produced novel synthetic nucleotides of notifiable dengue virus 1-4, Japanese encephalitis, yellow fever and Zika flaviviruses using OE-PCR is useful in understanding the dynamics of flaviviral species and holds potential for the development of synthetic nucleotide-based immunogens.
\end{abstract}

Lay abstract: Dengue virus (1-4), Japanese encephalitis and Zika fevers are notable mosquito-borne infections that continue to be problematic in the Philippines and its southeast Asian neighbors. Flaviviral yellow fever affects other tropical countries. This study aimed to effectively and efficiently synthesize flaviviral nucleotides through a predictively accurate algorithmic overlap extension-polymerase chain reaction design. The cost-saving arithmetic formulation has enabled the advanced production of 253-256 bp flaviviral nucleotide lengths. The newly synthesized and sequenced products are now ready for further experimentation and open the door for immunological exploration.

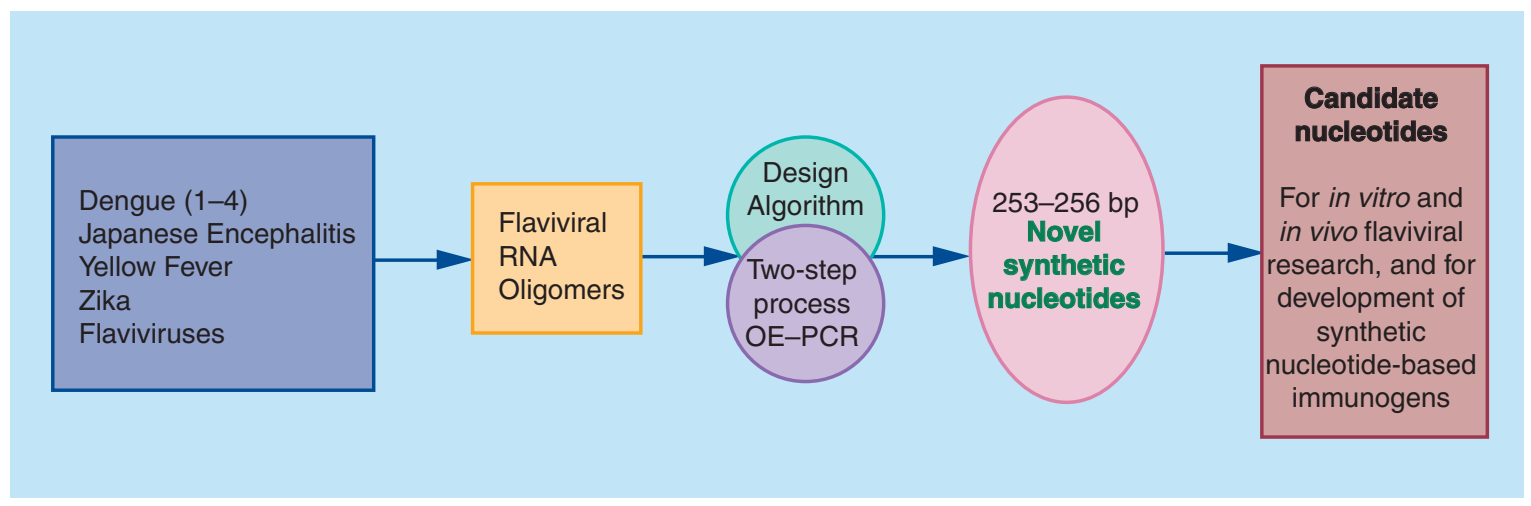

First draft submitted: 28 August 2018; Accepted for publication: 27 September 2018; Published online: 13 November 2018

Keywords: algorithm • flaviviruses • oligomer • overlap-extension PCR • synthetic nucleotides 


\begin{tabular}{|c|c|c|c|c|}
\hline Coding number & Flavivirus species & Accession number & Start & End \\
\hline 2 & Dengue virus 2 & NC_001474.2 & 9157 & 9388 \\
\hline 3 & Dengue virus 4 & NC_002640.1 & 9150 & 9381 \\
\hline 5 & Dengue virus 1 & NC_001477.1 & 9158 & 9389 \\
\hline 8 & Dengue virus 3 & NC_001475.2 & 9149 & 9380 \\
\hline 15 & Zika virus & NC_012532.1 & 9245 & 9479 \\
\hline 25 & Yellow fever virus & NC_002031.1 & 9227 & 9461 \\
\hline 27 & Japanese encephalitis virus & NC_001437.1 & 9270 & 9504 \\
\hline
\end{tabular}

Dengue and Zika fevers, including Japanese encephalitis (JEV), are particularly burdensome mosquito-borne infections in the Philippines and are also selectively reported in Japan [1-4]. The challenges these diseases pose and the need to efficiently convert these notable flaviviruses into larger DNA fragments are evident, considering that the performance of currently developed vaccines and DNA-based immunogens remain imperfect [5,6]. Molecularbased methodologies are presently available in the literature with varying degrees of precision and accuracy for PCR amplification, disease detection, genetic probing and DNA construction $[7,8]$. Many artificial genes are now commercially produced by several manufacturers. Moreover, the inherent risks, including the biosecurity concerns associated with handling and producing DNA fragments of pathogenic viruses, as well as some highly virulent bacteria, delimit its commercialized propagation $[4,9,10]$. Depending on the type of oligomer being used, the costeffectiveness of oligomer-size fragments varies. One of the promising modes for developing larger molecules of DNA is through the use of the overlap-extension polymer chain reaction (OE-PCR) method, which produces DNA fragments larger than $100 \mathrm{bp}$ in size using synthetic oligomers $[9,11,12]$. The concerns that may have contributed to a rise in the price of oligomers have encouraged the development of novel model templates for PCR primers that can serve as a new application tool for OE-PCR. However, the oligomer design for OE-PCR may be problematic. This is because of homogeneity of the annealing temperature of the overlapping regions as well as the sense-antisense design of sequences [11,12]. As for positive controls for PCR detection, many DNA fragments may be produced; therefore, an algorithmic or automated design of oligomers for OE-PCR is indispensable [12]. Using an algorithmic OE-PCR design, this study was performed to efficiently propagate synthetic nucleotides of select flaviviruses $[3,4]$. Nucleotides of notifiable flaviviruses, namely dengue (1-4), JEV, yellow fever (YF) and Zika fevers, were efficiently and cost-effectively synthesized without the use of a DNA template, hence, generating synthetic select flaviviral DNAs useful for further viral studies and immunological explorations.

\section{Materials \& methods}

The select list of notifiable flaviviral pathogens

Table $1 \&$ Box 1 show the profiles of the seven selected flaviviruses (Eurofins Genomics, Co. Limited, Ota-ku Tokyo, Japan) for amplification using OE-PCR.

The OE-PCR in this experiment was performed via two processes: the first one is using the reference or original OE-PCR protocol [11,12] and the next one is the modified version of the OE-PCR. This was done to ensure optimization of deriving the desired amplified products.

\section{Reference/original overlap extension-polymerase chain reaction}

Preparation of the first-stage primer mixed solution

(A) Both ends primer $(1,6) 2.5 \mu \mathrm{l}$

(B) Central primer (2-5) $0.5 \mu \mathrm{l}$

(U) Deionized water (DW) $18 \mu \mathrm{l}$

Preparation of reaction solution for the first-step PCR

(A) First-stage primer mixed solution $\times 6 \mu \mathrm{l}$

(A) GoTaq $12.5 \mu \mathrm{l}$

(U) DW $6.5 \mu \mathrm{l}$ 
Box 1. The oligomers of the flaviviruses (dengue virus 1-4, Japanese encephalitis, yellow fever and Zika).

flavi|2|1: ATGTATGCCGATGACACCGCAGGATGGGATACAAGAATCACACTAGAAGACCTAAAAAAT: 60 flavi|2|2: TTGTGTTCTCCTTCCATGTGGTTTGTTACCATTTCTTCATTTTTTAGGTCTTCTAGTGT: 59

flavi |2|3: CATGGAAGGAGAACACAAGAAACTAGCCGAGGCCATTTTCAAACTAACGTACCAAAACA: 59

flavi |2|4: TGTGCCTCTTGGTGTTGGTCTTTGCACACGCACCACCTTGTTTTGGTACGTTAGTTTG: 58

flavi |2|5: CAACACCAAGAGGCACAGTAATGGACATCATATCGAGAAGAGACCAAAGAGGTAGTGGA: 59

flavi |2|6: TGGTGAAAGTATTGAGTCCATAGGTGCCAACTTGTCCACTACCTCTTTGGTCTC: 54

flavi |3|1: ATGTATGCTGATGACACAGCAGGCTGGGACACAAGAATCACTGAGGATGACCTTCAAA: 58

flavi |3|2: TGGTGGGGAGCCATCTGTTCCGTGATCAGTTCCTCATTTTGAAGGTCATCCTCAGT: 56

flavi |3|3: TGGCTCCCCACCACAAGATCCTAGCCAAAGCCATTTTCAAACTAACCTATCAAAACAA: 58

flavi |3|4: TCCCCGCGGTGTGGGTCTGAGGACTTTCACCACTTTGTTTTGATAGGTTAGTTTG: 55

flavi |3|5: CACCGCGGGGAGCGGTGATGGATATCATATCCAGGAAAGACCAAAGAGGTAGTGGA: 56

flavi |3|6: TGGTGAATGTGTTCAAACCATATGTTCCAACTTGTCCACTACCTCTTTGGTCTT: 54

flavi |5|1: ATGTATGCAGATGACACAGCCGGATGGGACACAAGAATAACAGAGGATGATCTTCAGAA: 59

flavi |5|2: CATGTTCAGGTTCCATGATGTCAGTGATTTTGGCCTCATTCTGAAGATCATCCTCTGT: 58

flavi |5|3: CATGGAACCTGAACATGCCCTATTGGCCACGTCAATCTTTAAGCTAACCTACCAAAACA: 59

flavi |5|4: GTTCCATTTTTCGCTGGTCTCTGCACCCTTACTACCTTGTTTTGGTAGGTTAGCTTA: 57

flavi |5|5: AGCGAAAAATGGAACCGTGATGGATGTCATATCCAGACGTGACCAGAGAGGAAGTGG: 57

flavi |5|6: TGGTGAAGGTGTTTAAGCCATAGGTTCCAACCTGTCCACTTCCTCTCTGGTCA: 53

flavi |8|1: ATGTATGCTGATGACACAGCTGGTTGGGACACAAGAATAACAGAAGATGACCTGCACA: 58

flavi |8|2: TGTGTTCAGGGTCCATTTGCTGTATGATCTTTTCCTCATTGTGCAGGTCATCTTCTG: 57

flavi |8|3: AATGGACCCTGAACACAGGCAGTTAGCGAACGCTATATTCAAGCTCACATACCAAAACA: 59

flavi |8|4: TGCCCGTTGGAGTCGGTCGTTGAACTTTGACCACTTTGTTTTGGTATGTGAGCTTG: 56

flavi |8|5: ACTCCAACGGGCACGGTAATGGATATTATATCTAGGAAAGACCAAAGGGGCAGT: 54

flavi |8|6: TGGTGAATGTATTCAGGCCATAAGTTCCCAGTTGTCCACTGCCCCTTTGGTC: 52

flavi |15|1: ATGTACGCAGATGACACTGCTGGCTGGGACACCCGCATTAGTAAGTTTGATCTGGAGAA: 59

flavi |15|2: GTGCCCTTCCTCCATTTGGTTGGTAATCAGAGCTTCATTCTCCAGATCAAACTTACT: 57

flavi |15|3: TGGAGGAAGGGCACAGAACTCTGGCGTTGGCCGTGATTAAATACACATACCAAAACAA: 58

flavi |15|4: TTTTTCCTCCTTCAGCTGGTCTGAGAACCTTCACCACTTTGTTTTGGTATGTGTATTTA: 59

flavi |15|5: CAGCTGAAGGAGGAAAAACAGTTATGGACATCATTTCAAGACAAGACCAGAGAGGGAG: 58

flavi |15|6: TGGTGAATGTGTTGAGAGCATAAGTGACAACTTGTCCACTCCCTCTCTGGTCTTGTC: 57

flavi |25|1: TTCTACGCGGATGACACCGCTGGATGGGACACGCGCATCACAGAGGCAGACCTTGAT: 57

flavi |25|2: TGTGATGTGGGCTCATGTAGTTCAAGATCTCCTGTTCATCATCAAGGTCTGCCTCTG: 57

flavi |25|3: ATGAGCCCACATCACAAAAAACTGGCACAAGCAGTGATGGAAATGACATACAAGAACA: 58

flavi |25|4: TTCCCTCCTGGGGCTGGTCTCAACACTTTCACCACTTTGTTCTTGTATGTCATTTCC: 57

flavi |25|5: GCCCCAGGAGGGAAAGCCTACATGGATGTCATAAGTCGACGAGACCAGAGAGGAT: 55

flavi |25|6: TGGTGATGGTGTTCAGAGCATAAGTCACTACCTGCCCGGATCCTCTCTGGTCTCGT: 56

flavi |27|1: ATGTACGCTGATGACACCGCCGGATGGGACACTAGAATTACCAGAACTGATTTAGAAAA: 59

flavi |27|2: GGTGTTCACCGTCTAGGAGCTCCAGCACCTTGGCTTCATTTTCTAAATCAGTTCTGGT: 58

flavi |27|3: CCTAGACGGTGAACACCGCATGCTCGCTCGAGCCATAATTGAACTGACTTACAGGCACA: 59

flavi |27|4: CTTTCCTTCTGCTGCAGGTCTCATGACCTTGACCACTTTGTGCCTGTAAGTCAGTTCA: 58

flavi |27|5: TGCAGCAGAAGGAAAGACCGTAATGGACGTGATATCAAGAGAAGATCAAAGGGGGAG: 57

flavi |27|6: TCGTGAAAGTGTTAAGGGCATAAGTGACCACCTGTCCACTCCCCCTTTGATCTTCT: 56

First-stage PCR reaction

(A) $95^{\circ} \mathrm{C} 2 \min 21$

(C) $72^{\circ} \mathrm{C}-2 \mathrm{~min}$

Preparation of the second-step PCR solution

(A) First-step PCR reaction solution $1 \mu \mathrm{l}$

(B) Both ends primer (stock solution) $1 \mu \mathrm{l} \times 2$

(C) GoTaq $12.5 \mu \mathrm{l}$

(D) $9.5 \mu \mathrm{l}$ of DW

Second-step PCR reaction

(A) $95^{\circ} \mathrm{C} 2 \mathrm{~min}$

(A) $\left(95^{\circ} \mathrm{C}-1 \mathrm{~min} \rightarrow 72^{\circ} \mathrm{C}-2 \mathrm{~min}\right) \times 35$-times

(C) $72^{\circ} \mathrm{C}-2 \mathrm{~min}$ 
Start of input data: nucleotide sequence/s (Flaviviral species)

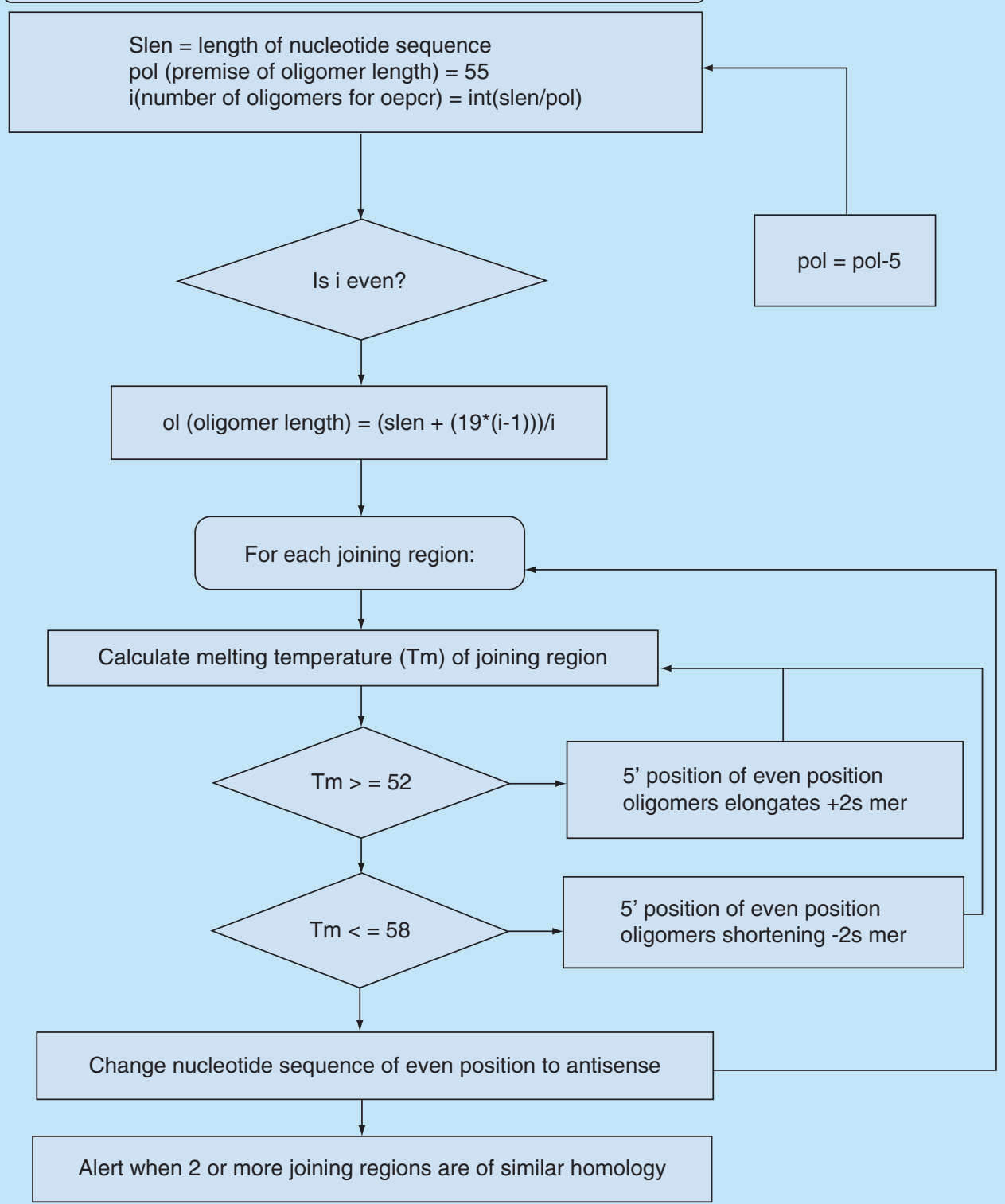

Figure 1. Overlap extension-polymerase chain reaction design algorithm.

This formulation was prepared to algorithmically plan and accurately calculate the desired product for OE-PCR. The process was initially done using manual algorithm whereby executable OE-PCR oligomers can be designed on the limitation of user-determined oligomer length. The platform made use of Linux (Ubuntu). OE-PCR: Overlap extension-polymerase chain reaction.

Modified overlap extension-polymerase chain reaction (OE-PCR)

1. Preparation of the first-stage primer-mixed solution
(A) Both ends primer $(1,6) 2.5 \mu \mathrm{l}$
(B) Central primer (2-5) $0.5 \mu \mathrm{l}$
(U) DW $193 \mu \mathrm{l}$
(A) GoTaq $12.5 \mu \mathrm{l}$
(U) DW $6.5 \mu \mathrm{l}$ 
(A)

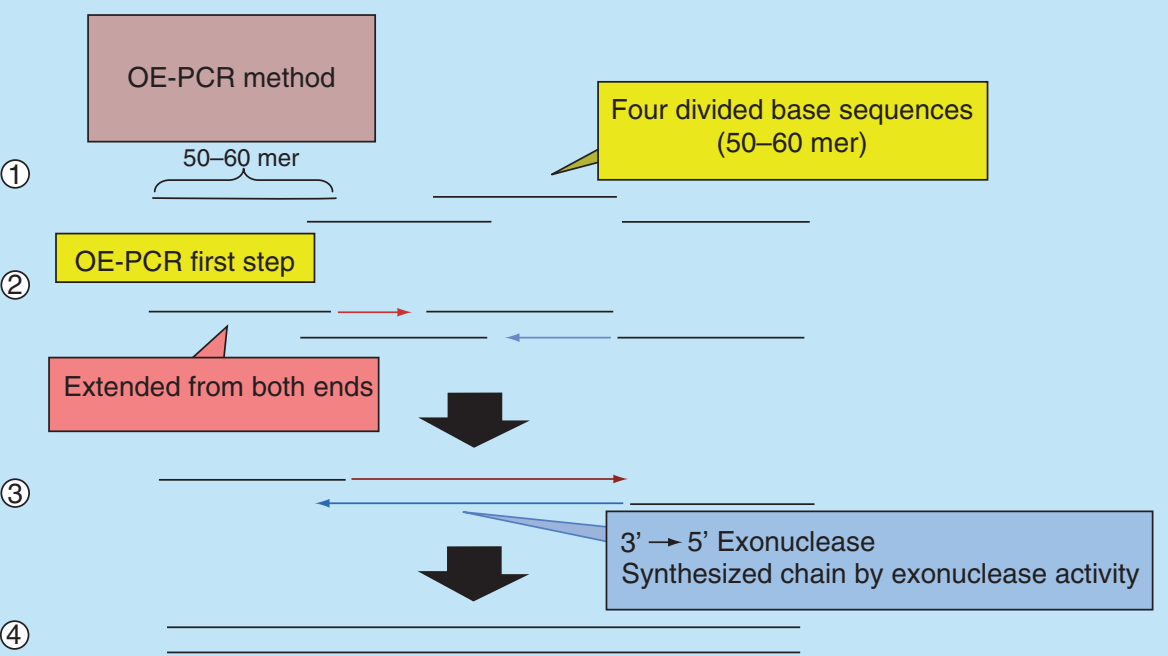

(B)
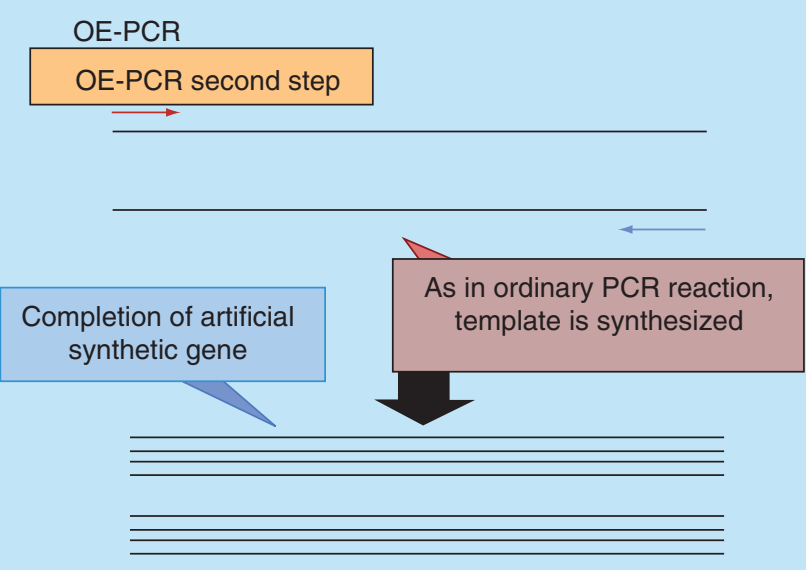

Figure 2. Diagram of the two-step cycle overlap extension-polymerase chain reaction (A: first step; B: second step). 1st PCR: For OE-PCR, 50-60mer oligomers were designed to have 19-23mer overlapping regions and make partial dimers (a). At the start of extension step of $1^{\text {st }}$ PCR, DNA was synthesized by polymerase which has a $3^{\prime} \rightarrow 5^{\prime}$ exonuclease activity (b). From the exonuclease activity, some oligomers were digested and replaced by synthesized DNA strand (c). Whole length of DNA was synthesized (d).

OE-PCR: Overlap extension-polymerase chain reactions.

2. First-stage PCR reaction
(A) $95^{\circ} \mathrm{C} 2 \mathrm{~min}$
(A) $\left(95^{\circ} \mathrm{C}-1 \mathrm{~min} \rightarrow 48^{\circ} \mathrm{C}-1 \mathrm{~min} \rightarrow 72^{\circ} \mathrm{C}-1 \mathrm{~min}\right) \times 25$
(C) $72^{\circ} \mathrm{C}-2 \mathrm{~min}$

3. Preparation of the second-step PCR solution
(A) First-step PCR reaction solution $1 \mu \mathrm{l}$
(B) Both ends primer (stock solution) $1 \mu \mathrm{l} \times 2$
(C) GoTaq $12.5 \mu \mathrm{l}$
(D) $9.5 \mu \mathrm{l}$ of DW 


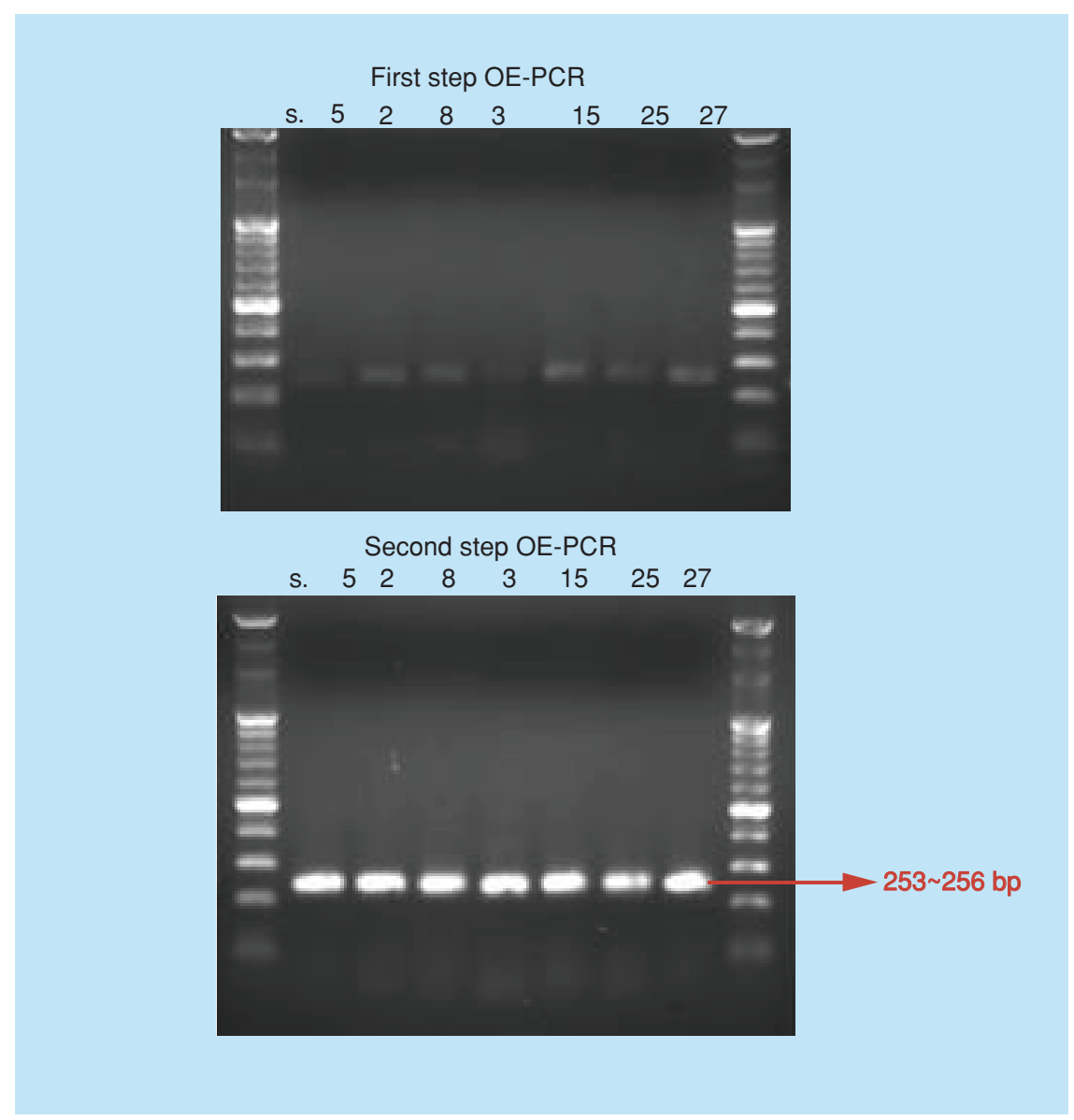

Figure 3. First and second step overlap extension-polymerase chain reaction showing prominent DNA bands. Note the almost similar product sizes of 253-256 bp. Each of the four Dengue virus has $253 \mathrm{bp}$; Zika, yellow fever and Japanese encephalitis has 256 bp DNAs. OE-PCR: Overlap extension-polymerase chain reactions.

4. Second-step PCR reaction
(A) $95^{\circ} \mathrm{C} 2 \mathrm{~min}$
(A) $\left(95^{\circ} \mathrm{C}-1 \mathrm{~min} \rightarrow 72^{\circ} \mathrm{C}-2 \mathrm{~min}\right) \times 25$
(C) $72^{\circ} \mathrm{C}-2 \mathrm{~min}$

Depicted in Figure 1 is the principle and process of OE-PCR as applied in this experiment.

In the second PCR, the most outside oligomers work for PCR primers and amplified the whole size of target DNA.

An electrophoresis of OE-PCR products was done for PCR product-size prediction after amplification of both OE-PCR and pathogen-specific PCR. Single product was confirmed on $1.5 \%$ agarose gel electrophoresis with $1 \times$ Tris/Borate/EDTA (TBE) buffer and UV-visualized with $0.7 \mathrm{micro}-\mathrm{g} / \mathrm{ml}$-ethidium bromide (Figure 2). The PCR product sizes were determined by comparison with DNA molecular weight marker whereby sizes of the products estimated are referred to the 100 bp-ladder (Dooh Chemical Co., Ltd, Sapporo, Japan).

\section{Results \& discussion}

Stepwise performance of overlap extension-polymerase chain reaction

The following notable flaviviruses, namely dengue (4 types), YF, JEV and Zika, were specifically processed for production of artificial DNAs. The algorithmic formulated OE-PCR was subsequently carried out in two-step cycles, with each cycle's DNA products depicted via UV-illuminated bands following gel electrophoresis (Figure 3). In the preliminary trials using this algorithmic OE-PCR, it was observed that a high success rate was derived when the number of oligomers was six or less compared with using an 8-oligomer-set or more. Thus, when OE-PCR 


\begin{tabular}{|c|c|c|c|c|}
\hline Number & $\begin{array}{l}\text { DDBJ/NCBI gene bank } \\
\text { accession number }\end{array}$ & $\begin{array}{l}\text { Flavivirus } \\
\text { species }\end{array}$ & $\begin{array}{l}\text { Complete } \\
\text { genome }\end{array}$ & Nucleotide sequence (synthetic) \\
\hline 1 & LC227563 & Dengue virus 1 & 9158-9410 & $\begin{array}{l}\text { ATGTATGCAGATGACACAGCCGGATGGGACACAAGAATAACAGAGGATGATCTTCAGAATGA } \\
\text { GGCCAAAATCACTGACATCATGGAACCTGAACATGCCCTATTGGCCACGTCAATCTTTAAGCTA } \\
\text { ACCTACCAAAACAAGGTAGTAAGGGTGCAGAGACCAGCGAAAAATGGAACCGTGATGGATG } \\
\text { TCATATCCAGACGTGACCAGAGAGGAAGTGGACAGGTTGGAACCTATGGCTTAAACACCTTCA } \\
\text { CCA }\end{array}$ \\
\hline 2 & LC227561 & Dengue virus 2 & 9157-9409 & $\begin{array}{l}\text { ATGTATGCCGATGACACCGCAGGATGGGATACAAGAATCACACTAGAAGACCTAAAAAAATGA } \\
\text { AGAAATGGTAACAAACCACATGGAAGGAGAACACAAGAAACTAGCCGAGGCCATTTCAAAA } \\
\text { CTAACGTACCAAAACAAGGTGGTGCGTGTGCAAAGACCAACACCAAGAGGCACAGTAATGG } \\
\text { ACATCATATCGAGAAGAGACCAAAGAGGTAGTGGACAAGTTGGCACCTATGGACTCAATACT } \\
\text { TTCACCA }\end{array}$ \\
\hline 3 & LC227562 & Dengue virus 3 & 9149-9401 & $\begin{array}{l}\text { ATGTATGCTGATGACACAGCTGGTTGGGACACAAGAATAACAGAAGATGACCTGCACAATGA } \\
\text { GGAAAAGATCATACAGCAAATGGACCCTGAACACAGGCAGTTAGCGAACGCTATATTCAAGC } \\
\text { TCACATACCAAAACAAAGTGGTCAAAGTTCAACGACCGACTCCAACGGGCACGGTAATGGAT } \\
\text { ATTATATCTAGGAAAGACCAAAGGGGCAGTGGACAACTGGGAACTTATGGCCTGAATACATTC } \\
\text { ACCA }\end{array}$ \\
\hline 4 & LC227568 & Dengue virus 4 & 9150-9402 & $\begin{array}{l}\text { ATGTATGCTGATGACACAGCAGGCTGGGACACAAGAATCACTGAGGATGACCTTCAAAATGA } \\
\text { GGAACTGATCACGGAACAGATGGCTCCCACCACAAGATCCTAGCCAAAGCCATTTTCAAACT } \\
\text { AACCTATCAAAACAAAGTGGTGAAAGTCCTCAGACCCACACCGCGGGGAGCGGTGATGGATA } \\
\text { TCATATCCAGGAAAGACCAAAGAGGTAGTGGACAAGTTGGAACATATGGTTTGAACACATTC } \\
\text { ACCA }\end{array}$ \\
\hline 5 & LC227560 & $\begin{array}{l}\text { Japanese } \\
\text { encephalitis } \\
\text { virus }\end{array}$ & $9270-9525$ & $\begin{array}{l}\text { ATGTACGCTGATGACACCGCCGGATGGGACACTAGAATTACCAGAACTGATTTAGAAAATGAA } \\
\text { GCCAAGGTGGTGGAGCTCCTAGACGGTGAACACCGCATGCTCGCTCGAGCCATAATTGAACTG } \\
\text { ACTTACAGGCACAAAGTGGTCAAGGTCATGAGACCTGCAGCAGAAGGAAAGACCGTAATGG } \\
\text { ACGTGATATCAAGAGAAGATCAAAGGGGGAGTGGACAGGTGGTCACTTATGCCCTTAACACT } \\
\text { TTCACGA }\end{array}$ \\
\hline 6 & LC227567 & $\begin{array}{l}\text { Yellow fever } \\
\text { virus }\end{array}$ & 9227-9482 & $\begin{array}{l}\text { TTCTACGCGGATGACACCGCTGGATGGGACACGCGCATCACAGAGGCAGACCTTGATGATGAA } \\
\text { CAGGAGATCTTGAACTACATGAGCCCACATCACAAAAAACTGGCACAAGCAGTGATGGAAAT } \\
\text { GACATACAAGAACAAAGTGGTGAAAGTGTTGAGACCAGCCCCAGGAGGGAAAGCCTACATG } \\
\text { GATGTCATAAGTCGACGAGACCAGAGAGGATCCGGGCAGGTAGTGACTTATGCTCTGAACAC } \\
\text { CATCACCA }\end{array}$ \\
\hline 7 & LC227589 & Zika virus & $9245-9500$ & $\begin{array}{l}\text { ATGTACGCAGATGACACTGCTGGCTGGGACACCCGCATTAGTAAGTTTGATCTGGAGAATGAA } \\
\text { GCTCTGATTACCAACCAAATGGAGGAAGGGCACAGAACTCTGGCGTTGGCCGTGATTAAATAC } \\
\text { ACATACCAAAACAAAGTGGTGAAGGTTCTCAGACCAGCTGAAGGAGGAAAAACAGTTATGG } \\
\text { ACATCATTTCAAGACAAGACCAGAGAGGGAGTGGACAAGTTGTCACTTATGCTCTCAACACAT } \\
\text { TCACCA }\end{array}$ \\
\hline
\end{tabular}

DDBJ: DNA Data Bank of Japan; NCBI: National Center for Biotechnology Information.

product was used as a positive control, the ability to design PCR primers that produce about 100 bases in the product is required. This is possible at around $250 \mathrm{bp}$ long and with algorithmic precision a well-synthesized 253-256 bp of DNA bands was yielded.

DNA sequencing was carried out in similar steps as previously described [1,7] (Eurofins Genomics, Co. Ltd, Ota-ku Tokyo). The newly sequenced products were subsequently registered at DDBJ/NCBI Gene Bank (see Table 2).

Approaches to determine efficiency and cost-effectiveness of the current algorithmic OE-PCR tool versus the existing programs are presented in Table 3. These data delineate the utilized platform, the license, the program execution, the problems encountered in oligomer design and the methods of assembly [13-18].

It must be noted that the price of oligomers varies greatly depending on the length in base and according to the viral species involved. Owing to this, an algorithm was designed to achieve the maximum length within the lowest cost range in base size. Unfortunately, this is difficult to realize using the currently available reported design algorithms. In the stated design algorithms, the difficulty lies in how to control the length of the oligomer. This is because the setting of overlapping region of the oligomer has been designed first. With the current study, the basic length has been decided to begin with; coupled with a lowered priority on the thermodynamic stability of the overlapping region. This has resulted in fewer problems in the conduct of OE-PCR. 
Table 3. Comparative features of oligomer design and assembly methods for overlap extension-polymerase chain

\begin{tabular}{|c|c|c|c|c|c|c|}
\hline Program name & Study (year) & Platform & License & Execute & Problem & Ref. \\
\hline \multirow[t]{2}{*}{ Speedy genes } & $\begin{array}{l}\text { Currin A, Swainston N, Day P, \& Kell D. } \\
\text { SpeedyGenes: exploiting an improved } \\
\text { gene synthesis method for the efficient } \\
\text { production of synthetic protein libraries } \\
\text { for directed evolution. In Synthetic DNA } \\
\text { Humana Press, New York, NY, 63-78 } \\
\text { (2017) }\end{array}$ & $\begin{array}{l}\text { Web (http://g.gene-genie.app } \\
\text { spot.com/?) }\end{array}$ & Free & OK & $\begin{array}{l}\text { Design limitation } \\
\text { from AA sequence }\end{array}$ & [13] \\
\hline & $\begin{array}{l}\text { Kalendar R, Lee D and Schulman A. Java } \\
\text { web tools for PCR, in silico PCR and } \\
\text { oligonucleotide assembly and analysis. } \\
\text { Genomics 98(2), 137-144 (2011) } \\
\text { Kalendar R, Tselykh T, Khassenov B, \& } \\
\text { Ramanculov E. Introduction on using } \\
\text { the FastPCR software and the related } \\
\text { Java web tools for PCR and } \\
\text { ligonucleotide assembly and analysis. } \\
\text { PCR: Methods and Protocols, 33-64 } \\
\text { (2017) }\end{array}$ & $\begin{array}{l}\text { Windows/MacOS/Linux/Web } \\
\text { (http://primerdigital.com/fast } \\
\text { pcr.html) }\end{array}$ & $\begin{array}{l}\text { Free (web) } \\
\text { /charge }\end{array}$ & $\begin{array}{l}\text { OE-PCR oligomer } \\
\text { can be designed }\end{array}$ & $\begin{array}{l}\text { Some oligomer } \\
\text { exceeds its size }\end{array}$ & [14] \\
\hline SITICHER2.0 & $\begin{array}{l}\text { O'Halloran D, Uriagereka-Herburger I } \\
\text { and Bode K. STITCHER 2.0: primer } \\
\text { design for overlapping PCR } \\
\text { applications. Sci. Rep. 7:45349 (2017) }\end{array}$ & $\begin{array}{l}\text { Web } \\
\text { (http://www.ohalloranlab.net } \\
\text { /STITCHER_2_0/index.html) }\end{array}$ & Free & $\begin{array}{l}\text { Mainly for } \\
\text { designing } \\
\text { PCR-primers }\end{array}$ & $\begin{array}{l}\text { No function for } \\
\text { OE-PCR }\end{array}$ & [16] \\
\hline Primer mapper & $\begin{array}{l}\text { O'Halloran D. PrimerMapper: high } \\
\text { throughput primer design and } \\
\text { graphical assembly for PCR and SNP } \\
\text { detection. Sci. Rep. 6:20631 (2016) }\end{array}$ & Linux (Perl) & Free & $\begin{array}{l}\text { Mainly for } \\
\text { designing } \\
\text { PCR-primers }\end{array}$ & $\begin{array}{l}\text { No function for } \\
\text { OE-PCR }\end{array}$ & [17] \\
\hline $\begin{array}{l}\text { RGU-design } \\
\text { OE-PCR }\end{array}$ & $\begin{array}{l}\text { Camer and Endoh et al. } 2018 \text { Novel } \\
\text { synthetic DNAs of notifiable dengue, } \\
\text { Zika and other flaviviruses: algorithmic } \\
\text { OE-PCR }\end{array}$ & $\begin{array}{l}\text { Platform: Linux (Ubuntu) } \\
\text { (https://bitbucket.org/dendoh } \\
\text { _2/rgu-design-oepcr/src) }\end{array}$ & $\begin{array}{l}\text { Free (distribute on } \\
\text { BitBucket) }\end{array}$ & $\begin{array}{l}\text { OE-PCR oligomers } \\
\text { can be designed } \\
\text { on the limitation } \\
\text { of } \\
\text { user-determined } \\
\text { oligomer length. }\end{array}$ & $\begin{array}{l}\text { Program language } \\
\text { Ruby must be } \\
\text { installed on the } \\
\text { user's computer. } \\
\text { Skills for executing } \\
\text { Ruby program is } \\
\text { needed. }\end{array}$ & $\dagger$ \\
\hline
\end{tabular}

${ }^{\dagger}$ RGU-design OE-PCR refers to current report of Camer and Endoh et al., 2018 of Rakuno Gakuen University, Department of Radiation Biology, School of Veterinary Medicine, Japan. OE-PCR: Overlap extension-polymerase chain reaction.

\section{Conclusion}

The ongoing dilemma of largely problematic and imperfectly developed flaviviral immunogens prompted us to responsibly contribute on the need to develop an efficient algorithmic OE-PCR program design for synthetic nucleotide production of select notifiable dengue (1-4), JEV, YF and Zika flaviviruses. To this end, we developed an OE-PCR program that can be utilized as reference tool in the furtherance of flaviviral research. It can be deduced that the program formulation was found to be demonstrably reproducible, cost-effective and efficient in yielding substantial nucleotides of select notifiable flaviviral DNAs, which can be sourced out at the databases of DDBJ/NCBI with the respective accession numbers of LC227563, LC227561, LC227562, and LC227568 (dengue 1-4); LC227560 (JEV); LC227567 (YF); and LC227589 (Zika). The OE-PCR is a reliable method that can be utilized when designed and accurately predicted using algorithmic manipulation. Henceforth, we finally conclude that the DNA primer design using algorithmic OE-PCR is a valuable tool in producing synthetic nucleotide products of pathogenic species of flaviviruses.

\section{Future perspective}

The novel nucleotide sequences generated from this study can be utilized for studies looking to further understand the genomic diversity of a variety of flaviviral strains, including dengue (1-4), YF, JEV and Zika. In addition, clinical trials involving initial utilization of whole nucleotide sequence followed by booster immuno-stimulation of antigenic protein may serve as a starting point for generating long-term immune responses for flaviviral infections such as dengue and Zika, where trials for DNA-based vaccines are ongoing, yet faced with challenging dosedependency concerns. Further experiments should be conducted to determine whether administration of synthetic nucleotides in vivo would result in pathological or immunological response. 


\section{Authors' contributions}

GA Camer and D Endoh collaboratively conceptualized, conducted, and processed the research and its manuscript. Y Oikawa and $\mathrm{H}$ Omaki assisted in laboratory experimentation, provided inputs on graphics and facilitated in the manuscript presentation of GA Camer and D Endoh. GA Camer has been a visiting/guest professor in RGU, Japan. GA Camer wrote the manuscript with contributions from each author. All authors have read and agreed with the ultimate form of this manuscript.

Financial \& competing interests disclosure

This research was supported by the 'Development for the control strategy of vector-borne and zoonotic diseases in Okinawa, Japan and formulation of networking among stakeholders', which is part of Okinawa's Communicable Disease Research Hub Formation Promotion Project. Credit is also due to the funding support of the Guest Researcher Program of Rakuno Gakuen University, Japan.

The authors have no other relevant affiliations or financial involvement with any organization or entity with a financial interest in or financial conflict with the subject matter or materials discussed in the manuscript apart from those disclosed. No writing assistance was utilized in the production of this manuscript.

\section{Ethical conduct of research}

The authors state that they have obtained appropriate institutional review board approval or have followed the principles outlined in the Declaration of Helsinki for all human or animal experimental investigations. In addition, for investigations involving human subjects, informed consent has been obtained from the participants involved.

\section{Open access}

This work is licensed under the Creative Commons Attribution 4.0 License. To view a copy of this license, visit http://creativecomm ons.org/licenses/by/4.0

\section{Summary points}

- This project aimed to develop synthetic nucleotides using overlap extension-polymerase chain reaction (OE-PCR), particularly of flaviviral organisms that are notorious for their ability to cause both animal and human diseases.

- A method is provided for generating template designs that could help to address epidemics caused by select flaviviral dengue, Zika and related species.

- Expansion of OE-PCR as a tool in developing templates for viral studies is presented, addressing concern regarding the lack of reported commercial production of artificial nucleotides of these flaviviruses.

- This research has delivered a reproducibly effective and efficient algorithmic OE-PCR design that is suitable for yielding substantial nucleotides of these flaviviruses.

- These synthetic nucleotides could pave the way for future collaborative research exploring the possibility of generating synthetic flaviviral nucleotide-based immunogens.

\section{References}

1. Kutsuna S, Kato Y, Moi ML et al. Autochthonous dengue fever, Tokyo, Japan, 2014. Emerg. Infect. Dis. 21(3), 517-521 (2015).

2. Petronio J, Vinarao R, Flores K, Destura R. Continued circulation of a single genotype of dengue virus serotype 2 in the Philippines. Asian Pac. J. Trop. Med. 7(1), 30 (2014).

3. Huang Y, Higgs S, Horne K, Vanlandingham D. Flavivirus-mosquito interactions. Viruses 6 (11), 4703-4730 (2014).

4. Holbrook M. Historical perspectives on flavivirus research. Viruses 9(5), 1-97 (2017).

5. Normile D. Safety concerns derail dengue vaccination program. Science 358 (6370), 1514-1515 (2017).

6. Danko JR, Kochel T, Teneza-Mora N et al. Safety and immunogenicity of a tetravalent dengue DNA vaccine administered with a cationic lipid-based adjuvant in a phase clinical trial. Am. J. Trop. Med. Hyg. 98(3), 849-856 (2018).

7. Buchan B, Ledeboer N. Emerging technologies for the clinical microbiology laboratory. Clin. Microbiol. Rev. 27(4), 783-822 (2014).

8. Tee K, Wong T. Polishing the craft of genetic diversity creation in directed evolution. Biotechnol. Adv. 31(8), 1707-1721 (2013).

9. Kelwick R, MacDonald J, Webb T, Freemont P. Developments in the tools and methodologies of synthetic biology. Front. Bioeng. Biotechnol. 2, 60 (2014).

10. Howard J, Murashov V, Schulte P. Synthetic biology and occupational risk. J. Occup. Environ. Hyg. 14(3), 224-236 (2017).

11. Zhang P, Ding Y, Liao W, Chen Q, Zhang H, Qi P, Ren H. A simple, universal, efficient PCR-based gene synthesis method: sequential OE-PCR gene synthesis. Gene 524(2), 347-354 (2013). 
12. Horton RM, Cai Z, Ho SN, Pease LR. Gene splicing by overlap extension: tailor-made genes using the polymerase chain reaction. Biotechniques 54(3), 129-133 (2013).

13. Currin A, Swainston N, Day P, Kell D. SpeedyGenes: exploiting an improved gene synthesis method for the efficient production of synthetic protein libraries for directed evolution. Methods Mol. Biol. 147263-78 (2017).,

14. Kalendar R, Lee D, Schulman A. Java web tools for PCR, in-silico PCR and oligonucleotide assembly and analysis. Genomics 98(2), 137-144 (2011).

15. Kalendar R, Tselykh T, Khassenov B, Ramanculov E. Introduction on using the FastPCR software and the related java web tools for PCR and oligonucleotide assembly and analysis. Methods Mol. Biol. 162033-64 (2017).,

16. Kalendar R, Lee D, Schulman AH. FastPCR software for PCR, in-silico PCR and oligonucleotide assembly and analysis. In:DNA Cloning and Assembly Methods. Humana Press, NJ, USA, 271-302 (2014)

17. O'Halloran D, Uriagereka-Herburger I, Bode K. STITCHER 2.0: primer design for overlapping PCR applications. Sci. Rep. 7, 45349 (2017).

18. O'Halloran D. Primer Mapper: high throughput primer design and graphical assembly for PCR and SNP detection. Sci. Rep. 6, 20631 (2016). 